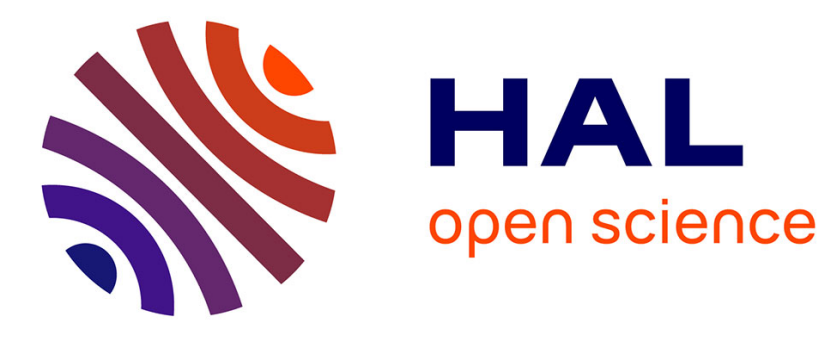

\title{
Kaleidoscope-based multi-view infrared system
}

Adrien Mas, Guillaume Druart, Maxime Vaché, Sylvain Favier, Aymeric

Alazarine, Eric Compain, Nathalie Morin, Nicolas Guérineau

\section{To cite this version:}

Adrien Mas, Guillaume Druart, Maxime Vaché, Sylvain Favier, Aymeric Alazarine, et al.. Kaleidoscope-based multi-view infrared system. Optics Letters, 2019, 44 (20), pp.4977-4980. 10.1364/OL.44.004977 . hal-02386569

\section{HAL Id: hal-02386569 https://hal.science/hal-02386569}

Submitted on 29 Nov 2019

HAL is a multi-disciplinary open access archive for the deposit and dissemination of scientific research documents, whether they are published or not. The documents may come from teaching and research institutions in France or abroad, or from public or private research centers.
L'archive ouverte pluridisciplinaire $\mathbf{H A L}$, est destinée au dépôt et à la diffusion de documents scientifiques de niveau recherche, publiés ou non, émanant des établissements d'enseignement et de recherche français ou étrangers, des laboratoires publics ou privés. 


\title{
Kaleidoscope-based multi-view infrared system
}

\author{
Adrien Mas, ${ }^{1,2}$ Guillaume druart, ${ }^{1}$ Maxime VachÉ, ${ }^{2}$ Sylvain FaVier, ${ }^{2}$ \\ Aymeric Alazarine, ${ }^{2}$ Eric Compain, ${ }^{2}$ Nathalie Morin, ${ }^{2}$ Nicolas Guerineau ${ }^{1}$
}

\author{
${ }^{1}$ Département Optique et Techniques Associées, ONERA, Université Paris Saclay F- 91123 Palaiseau, France \\ ${ }^{2}$ Bertin Technologies, 155 Rue Louis Armand ZI, 13290 Aix-en-Provence, France
}

Received XX Month XXXX; revised XX Month, XXXX; accepted XX Month XXXX; posted XX Month XXXX (Doc. ID XXXXX); published XX Month XXXX

\begin{abstract}
Multi-view snapshot systems are used for a wide range of applications in all the spectral ranges. We submit here the study and the realization of an optical system using a kaleidoscope in the Long Wavelength Infrared (LWIR) and compatible with uncooled infrared detectors such as microbolometers. The optical system has a high numerical aperture, a wide field of view and uses a single focal plane array. We establish here the advantages of this technology on other design strategies, its design rules for every subset of the optical architecture and present the results of a first demonstrator. (C) 2019 Optical Society of America
\end{abstract}

http://dx.doi.org/10.1364/OL.99.099999

Snapshot multi-view systems can manage advanced imagery functions [1]. Thus, they are considered for a wide range of applications such as, polarization, miniaturization, superresolution, 3D imagery and spectral imagery [2-3]. Different optical architectures have been proposed to meet these new imagery needs, exploring multi-aperture, single-aperture or hybrid concepts [4]. To cover a wide range of applications in the infrared spectral bandwidth, we are looking for a design with a good spatial resolution for pixel co-registration and fields of view ranging from 10 to 60 degrees. We intend to use uncooled infrared detectors, especially microbolometers, in the Long Wavelength Infrared (LWIR). The signal delivered by a microbolometer can easily drift with the environmental temperature. Therefore, for remote sensing applications, it is worth producing the images on a single Focal Plane Array (FPA) before processing them so each image drifts in the same way. In this paper, we will thus focus on infrared designs able to produce multiple images on a single FPA without mentioning systems based on array of cameras [1]. Moreover, using a microbolometer implies a high numerical aperture of the optical system to maximize the signal-to-noise ratio, typically F/1. One of the most classic designs for achieving multiple images on a single detector is the TOMBO multi-channel architecture [5] which has been widely studied. This concept uses an array of imaging elements such as a monolithic lenslet array, to juxtapose several optical systems and to produce these multiple images on a single FPA. A LWIR uncooled TOMBO camera, has already been made [6].
However, its major drawback relies on the relationship between the F-number $(F \#)$ and the field of view (FOV) to avoid blind areas on the FPA or field overlay between adjacent channels. Indeed, in order to maximize the number of useful pixels associated with a single lenslet of the ТОМВО system while having the lowest Fnumber, FOV (in radians) and F\# are linked by the following equation [7]:

$$
F O V=\frac{1}{F \#} .
$$

Validating Eq. (1) means that the lenses have the same size as their associated subimages. If we allow that the size of the lenses can be smaller than their subimages, Eq. (1) becomes $F \# \geq 1 / F O V$. Therefore, for a $\mathrm{FOV}$ of $10^{\circ}$, the F-number should be greater than 6 . This F-number is not compatible when using a microbolometer. In other words, with the requirement of a F-number of 1 , the FOV should be greater than $60^{\circ}$ for an optimal use of pixels and a smaller FOV will yield unused pixel areas on the FPA between the subimages, as observed in [6]. To overcome this constraint, Carles's team proposed a two-stage freeform TOMBO architecture allowing an increase of the numerical aperture by using off-axis optics to tilt the optical axis of each channel [8]. Freeform surfaces are used to correct the induced off-axis aberrations generated by this approach. With this design, the unused pixel area can be reduced for small field of view systems (in [8], the field of view is equal to $8^{\circ}$ ). However, freeform surfaces that can be different from one channel to another increase drastically the complexity of the optical system. Since unconventional lenses have to be made and aligned, this solution becomes difficult to apply for more than nine images. Finally, multichannel architectures must manage crosstalk between the different channels, usually by using walls, since a field stop is not easily implemented for high numerical aperture [9]. However, the grazing incidence of rays on these walls can lead to problematic parasitic reflections. We conclude from the state of the art that multi-aperture concepts have limitations for high aperture systems with narrow FOVs.

Light-field type cameras were studied too [10-11]. Among these different concepts, the single aperture light-field design based on a kaleidoscope does not suffer from the limitations of multi-aperture systems [12] and has a field stop that prevents crosstalk. The principle of this optical architecture is to place, between a first 
imaging lens and a re-imaging one, a kaleidoscope which is a tube made of 4 plane mirrors. A sketch of this design is given in Fig. 1 . An intermediate image is formed by the first lens at the entrance of the kaleidoscope. By reflection in the kaleidoscope, the cones of incident beams are divided into $\mathrm{N} \times \mathrm{N}$ cones with different orientations that form virtual copies of the image produced by the first lens at the intermediate image plane. This array of images is then focused by the second lens on the FPA. In [12], a diffuser is used at the entrance of the kaleidoscope to diffuse the cone of incident beams and send it on the mirrors of the kaleidoscope. So a large number of images are formed independently of the aperture of the front lens and off-the-shelf lenses can be used. However, a diffuser induces serious loss of light and image quality and this approach is thus not adapted for LWIR systems using an uncooled detector. In [12], authors also proposed a light-field imaging system without diffuser by adding a field lens next to the entrance of the kaleidoscope. As we will see later, if carefully designed, this approach is very interesting for efficient light gathering. However in [12], the use of off-the-shelf lenses makes this approach difficult and the authors use a long kaleidoscope resulting in a decrease of the numerical aperture of the whole system. In this paper we demonstrate that a kaleidoscope concept can be adapted to meet the light efficiency constraints of uncooled infrared detectors. We demonstrate the FOV- F\# uncoupling capacity of this system. Finally, we highlight the key dimensions of the architecture to ensure a good distribution of light between the different subimages while maintaining a good compactness thanks to a careful design of all the subsets: the front lens, the kaleidoscope and the re-imaging lens. As a consequence, custom-made optics have to be designed.

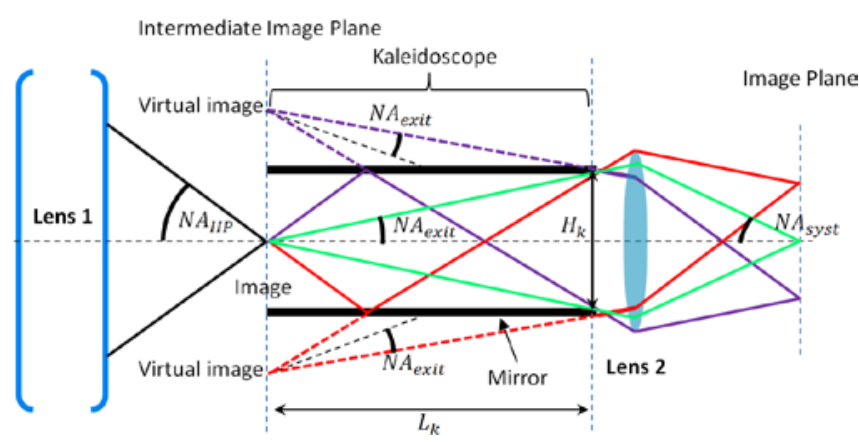

Fig. 1. Structure of the kaleidoscope architecture

The kaleidoscope is the central part of the design and impacts the dimensioning of the front lens and of the re-imaging lens for an optimal configuration. The front lens images a scene situated at infinity on an intermediate image plane at the entrance of the kaleidoscope. This way, the entrance section of the kaleidoscope is used as a field stop and its section defines the shape of the images. The presence of a field stop prevents crosstalk between the different subimages. A mask can be placed at the entrance of the kaleidoscope to limit grazing rays on the mirror. However the more the mask overflows on the intermediate image, the more unused pixels will appear between each image on the FPA. At first approximation, the entrance height $H_{k}$ of the kaleidoscope is linked to the system's field of view FOV (in radians) and to the focal length $f_{1}$ ' of the front lens by the following relation:

$$
H_{k} \cong f_{1}^{\prime} F O V \text {. }
$$

Without a diffuser at the entrance of the kaleidoscope, the front lens needs to have an adequate aperture in order to maximize the light gathering capability. To guarantee a uniform light distribution between the images, the numerical aperture at the intermediate image plane $N A_{I I P}$ should be divided inside the kaleidoscope into NxN numerical apertures with similar cone angles as shown Fig. 1. Then the numerical aperture at the exit of the kaleidoscope $N A_{\text {exit }}$ is given by the following equation:

$$
N A_{\text {exit }}=\frac{N A_{I I P}}{N} .
$$

As a consequence, to have a uniform light distribution between the images, the dimension of the kaleidoscope is linked to the numerical aperture at the intermediate image plane. Fig. 2 illustrates this condition. Rays contributing to a subimage are plotted in a dedicated color: green for the top image, blue for the bottom image and red for the central image.

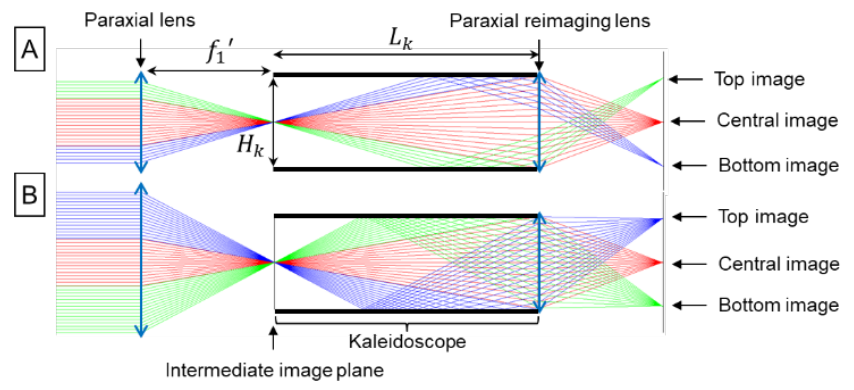

Fig. 2. Impact of the numerical aperture of the front lens, for the kaleidoscope's given dimension, on the formation of the image points for A) a numerical aperture undersized compared to the kaleidoscope dimension, B) an adapted numerical aperture.

Fig. 2.A shows an undersized numerical aperture of the front lens compared to the kaleidoscope's dimension. Indeed, images induced by a single reflection have less luminous flux than the central image. As a consequence, the numerical aperture on the intermediate image plane of height $H_{k}$ and the length $L_{k}$ of the kaleidoscope need to satisfy the following equation:

$$
N A_{I I P}=N \sin \left(\operatorname{atan}\left(\frac{H_{k}}{2 L_{k}}\right)\right) \approx N \frac{H_{k}}{2 L_{k}} .
$$

Fig. 2.B satisfies condition (4) and the light distribution is fairly uniform between the image points. The kaleidoscope system was designed to produce $3 \times 3$ images. A higher numerical aperture than condition (4) will add rays having double reflection on opposite mirrors and will produce additional unwanted images. It is clear from this figure that the entrance pupil is actually vertically divided into 3 sub-pupils, each associated with a subimage. In this case, the front lens is equivalent to an array of 3x3 imaging apertures.

Now we have to guarantee Eq. (3) over the field of view. In [13], S. Pacheco et al. optimize a telecentric front lens as illustrated in Fig. 3.A. For simplicity, we represent the front lens and the re-imaging lens as ideal paraxial lenses in ZEMAX software. The configuration of Fig. 3.A has a telecentric front lens and we notice that an extra image point appears, associated with black rays, due to a double reflection inside the kaleidoscope. The upper image in green, 
obtained through reflection on the bottom mirror, receives less light than its counterpart at the bottom in blue. With the telecentric front lens, the exit pupil is placed at infinity. In fact, to fulfill the condition of Eq. (3), the exit pupil of the front lens needs to be at the exit section of the kaleidoscope as illustrated in Fig. 3.B. In Fig. 3.B, we add a field lens in the intermediate image plane to place the exit pupil of the front lens at the exit of the kaleidoscope. In this case, we clearly see that the light is better distributed between the image points and we avoid the double reflections inside the kaleidoscope. This constraint of having an exit pupil after the image plane for an imaging lens is not common and a custom-made front lens has to be designed to guarantee a good image quality. We have therefore defined the design rules for an optimal distribution of light between sub-images.

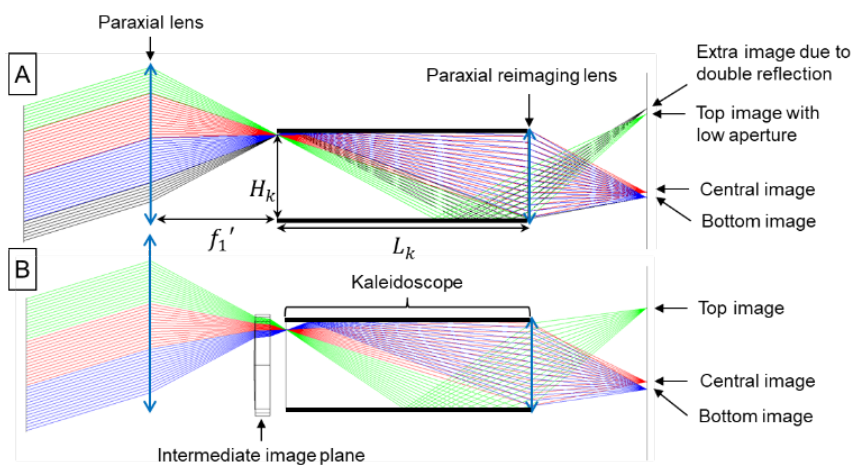

Fig. 3. Impact of the orientation of rays entering the kaleidoscope on the image formation in the field for A) a telecentric front lens, B) an exit pupil of the front lens in the exit plane of the kaleidoscope.

The re-imaging lens needs to re-image the array of images, having a height $N \times H_{k}$ in the intermediate image plane, on the FPA having a height $t_{d e t}$. This means that the re-imaging lens must guarantee the following magnification $M$ :

$$
M=\frac{t_{d e t}}{N H_{k}}
$$

Moreover, this re-imaging lens must have its entrance pupil at the exit of the kaleidoscope and must guarantee the numerical aperture of the whole system $N A_{\text {syst }}$ which is linked to the numerical aperture at the intermediate image plane and the features of the kaleidoscope. Combining Eq. (3) and Eq. (5) also gives an important kaleidoscope's feature:

$$
N A_{\text {syst }}=\frac{N A_{\text {exit }}}{M}=N A_{I I P} \frac{H_{k}}{t_{\text {det }}} .
$$

In our demonstrator, we decide that $t_{\text {det }}$ is equal to the height $H_{k}$ of the kaleidoscope. In that case, the re-imaging lens divides the focal length of each sub-image by a factor $N$ compared to the front lens focal length (Eq. (5)). However, the entrance pupil associated with each subimage is divided by $N$ by the kaleidoscope (Eq. (3)). Finally, a pixel would see the same optical etendue in the intermediate image plane as in the FPA and the numerical aperture of each subimage $N A_{\text {syst }}$ is equal to $N A_{I I P}$ as shown in Eq. (6). Sometimes, it could be easier to design a front lens with a lower numerical aperture, while keeping the numerical aperture and the FOV of the whole system constant. In that case, $H_{k}$ is greater than $t_{d e t}$ and Eq. (2) tells us that the focal length of the front lens increases. As a consequence, the size of the whole system increases.

Finally, by combining Eq. (6) with Eq. (4) and Eq. (2), by assuming that $t_{d e t}=H_{k}$ and by considering that the F-number of the whole system $\left(F \#_{\text {syst }}\right)$ is linked to $N A_{\text {syst }}$ by the following relation $F \#_{\text {syst }}=1 /\left(2 \times N A_{\text {syst }}\right)$, we obtain another feature of this system:

$$
F \#_{s y s t}=\frac{1}{F O V} \frac{L_{k}}{N f_{1}} .
$$

In comparison with Eq. (1) that describes an optimal TOMBO design, Eq. (7) shows that with a kaleidoscope system, the Fnumber is not directly linked with the FOV, which is an important property of this design.

Following the previously detailed design rules, we have designed a kaleidoscope-based optical system with the software ZEMAX. We wanted to limit as much as possible the number of optics and the number of aspheric surfaces to keep a simple manufacturing system. Thus, the system could easily be improved optically, to the detriment of manufacturing simplicity and optics alignment. We converge to the architecture illustrated in Fig. 4. It is opened at F/1 and has a field of view of $30^{\circ}$. The kaleidoscope has a square height of $9 \mathrm{~mm}$ wide and $25 \mathrm{~mm}$ long. The re-imaging lens has a magnification close to $1 / 3$ and the multi-imaging system produces $3 \times 3$ images. The central image is produced without reflection in the kaleidoscope; vertical and horizontal images are induced by a single reflection on the mirrors and diagonal images are induced by double reflection on orthogonal mirrors. The FPA chosen is an uncooled $640 \times 480$ detector with a pixel pitch of $25 \mu \mathrm{m}$. The total length of the optical system is $110 \mathrm{~mm}$ for a focal length of $6 \mathrm{~mm}$.

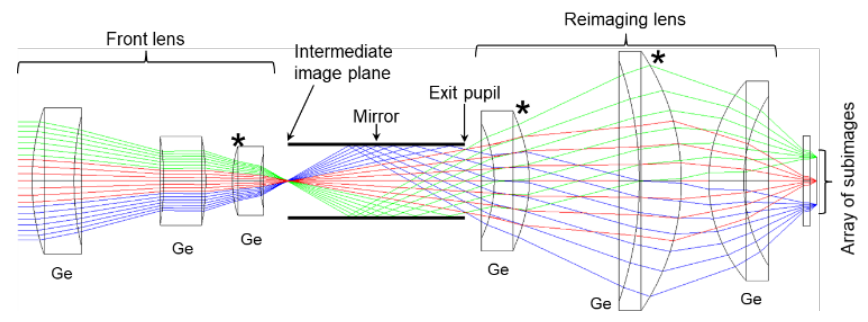

Fig. 4. Final infrared architecture based on a kaleidoscope and adapted for a microbolometer. Aspheric surfaces are marked with*.

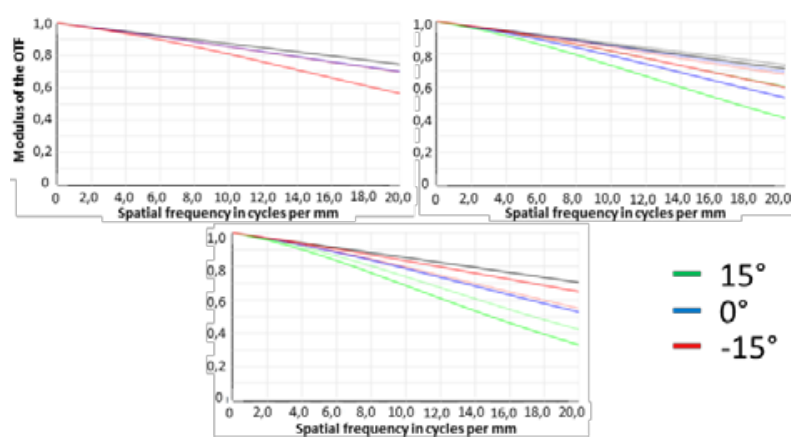

Fig. 5. Theoretical MTFs for different type of images and for 3 fields of view. On the left, the central image's MTF on the right, a side image's MTF; on the bottom, a diagonal image's MTF. 
In Fig. 4., the central field is represented to show the image points created. The theoretical polychromatic $(8 \mu \mathrm{m}-12 \mu \mathrm{m})$ Modulation Transfer Functions (MTFs) for different fields of view and for the central sub-image, a side sub-image and a diagonal sub-image are illustrated in Fig. 5. The image quality of the system is satisfactory for the 9 images for a $25 \mu \mathrm{m}$ pixel pitch FPA since the MTFs have contrast up to the Nyquist frequency at 20 cycles $/ \mathrm{mm}$.

Fig. 6 illustrates the light distribution between the sub-images. We see that it is almost the same between the central image and the side images but it is lower in the diagonal images. Indeed, we have dimensioned the kaleidoscope so that it is as compact as possible while distributing the light as well as possible on the vertical and horizontal images. However, the kaleidoscope is square-sectioned and our system is not optimized for the diagonal sub-images. The solution would be to increase the numerical aperture at the intermediate image plane to increase the luminous flux in diagonal images. Given the relation (4), the condition would become:

$$
N A_{I I P}=3 \sin \left(\operatorname{atan}\left(\frac{\sqrt{2} H_{k}}{2 L_{k}}\right)\right) .
$$

In our case, this increases drastically the numerical aperture and complicates the front objective. Indeed, a F-number equal to 0.75 is very difficult to obtain.

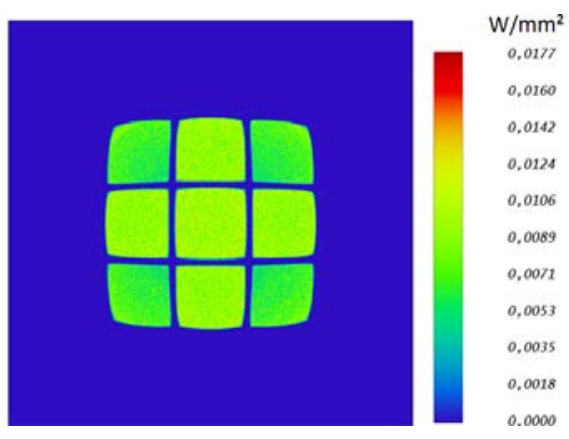

Fig. 6. Illustration of the distribution of 1 Watt of luminous flux between the different images of a system optimized for 5 images.

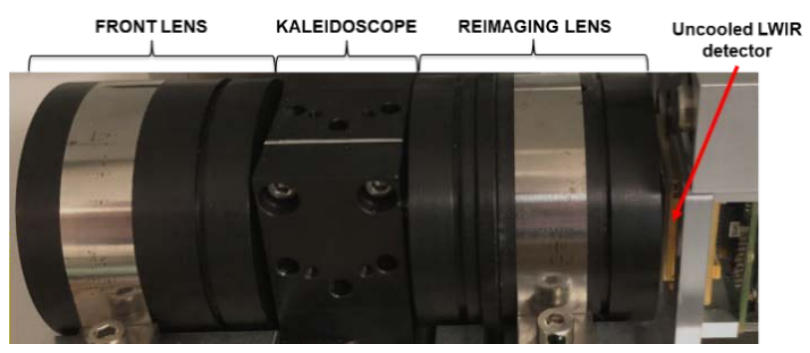

Fig. 7. Kaleidoscope prototype.

Based on the optical design of Fig. 4, a prototype has been made. It is shown in Fig. 7. Images are given in Fig. 8. We clearly see 3x3 sub-images; a central sub-image and 8 others with orientations that match one or two reflections inside the kaleidoscope.

We observe extra images resulting from double reflections on opposite mirrors which are due to an entrance pupil not perfectly defined. Thus, the numerical aperture of the rays entering the kaleidoscope is too high to avoid opposite double reflections.
The realization and the characterization of the prototype show that we have been able to adapt the kaleidoscope architecture to an application in the LWIR with an uncooled detector. Compared to a TOMBO design, this multi-imaging system has the following advantages. Firstly, it can answer to the high numerical aperture challenge while exploiting a maximum of pixels on a FPA by decoupling the field of view and the F-number. For this reason, it is particularly suited for systems requiring both a small field of view and a high aperture. Secondly, it has some flexibility to modify the field of view by only changing the front lens. Finally, there is no risk of crosstalk because the entrance of the kaleidoscope acts as a field stop. However, it is less compact than a TOMBO design but we have demonstrated that we can limit the size of the kaleidoscope by a careful design strategy that maximizes the light gathering efficiency. The kaleidoscope-based system uses conventional lenses and plane mirrors and thus it enables rapid and costeffective manufacturing and assembling. Different fields of application based on this kaleidoscope architecture can be explored such as spectral, polarization or 3D imaging.
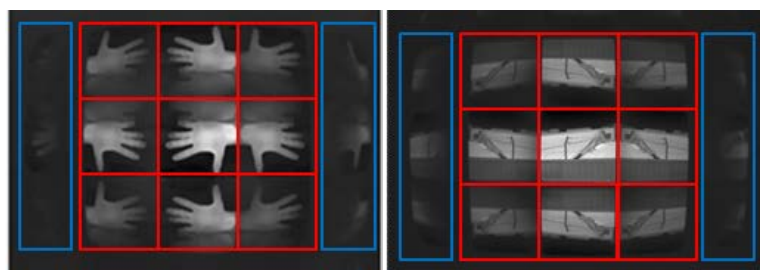

Fig. 8. On the left, an image of a hand through the kaleidoscope system which clearly shows orientations of each 9 images (framed in red); on the right, an image of a roof where we can distinguish small details like an overhead electrical wire that highlights the image quality of the system. Extra images resulting from double reflections on opposite mirrors are framed in blue.

\section{References}

1. B. Wilburn, N. Joshi, V. Vaish, E. Talvala, E. Antunez, A. Barth, A. Adams, M. Horowitz, M. Levoy, ACM Trans. Graph. 24 (3) pp. 765-776, (2005).

2. K. Venkataraman, D. Lelescu, J. Duparre, A. McMahon, G. Molina, P. Chatterjee, R. Mullis, S. Nayar, ACM Trans. Graph. 32(6), 13 (2013).

3. A. R. Harvey, G. Carles, L. Cowan, M. Preciado, J. Ralph, J. Babington, A. Wood, Proc. SPIE 10438, 1043806 (2017).

4. N. Hagen and M. W. Kudenov, Opt. Eng. 52, 090901 (2013).

5. J. Tanida, T. Kumagai, K. Yamada, S. Miyatake, K. Ishida, T. Morimoto, N. Kondou, D. Miyazaki, and Y. Ichioka, Appl. Opt. 40, 1806-1813 (2001).

6. A. Portnoy, N. Pitsianis, X. Sun, D. Brady, R. Gibbons, A. Silver, R. Te Kolste, C. Chen, T. Dillon, and D. Prather, Appl. Opt. 48, 2115-2126 (2009).

7. F. de la Barrière, G. Druart, N. Guérineau and J. Taboury, Appl. Opt. 50, 943-951 (2011).

8. G. Carles, G. Muyo, N. Bustin, A. Wood, A. R. Harvey, J. Opt. Soc. Am. A 32, 411-419 (2015).

9. G. Druart, N. Guérineau, R. Haïdar, E. Lambert, M. Tauvy, S. Thétas, S. Rommeluère, J. Primot,J. Deschamps, Proc. of SPIE Vol. 6992, 69920G, (2008).

10. K. Cossu, G. Druart, E. Belhaire, F. Champagnat, T. Lépine, Proc. SPIE 9948 99480B-1 (2016).

11. R. Horstmeyer, G. Euliss, R. Athale, and M. Levoy, Proceedings of the IEEE International Conference on Computational Photography (IEEE, 2009).

12. A. Manakov, J. F. Restrepo, O. Klehm, R. Hegedus, E. Eisemann, H. Seidel, and I. Ihrke, ACM Trans. Graph. 32, 47:1-47:14 (2013).

13. S. Pacheco, and R. Liang, Opt. Express 22, 16377-16385 (2014). 


\section{References}

1. B. Wilburn, N. Joshi, V. Vaish, E. Talvala, E. Antunez, A. Barth, A. Adams, M. Horowitz, M. Levoy, "High performance imaging using large camera arrays," ACM Transactions on Graphics 24(3), pp. 765-776, (2005).

2. K. Venkataraman, D. Lelescu, J. Duparre, A. McMahon, G. Molina, P. Chatterjee, R. Mullis, S. Nayar, "PiCam: An ultra-thin high performance monolithic camera array," ACM Transactions on Graphics 32(6), 13 (2013).

3. A.R. Harvey, G. Carles, L. Cowan, M. Preciado, J. Ralph, J. Babington, A. Wood, "The simplicity, complexity, and benefits of multi-aperture imaging in the thermal infrared," Proc. SPIE 10438, 1043806 (2017).

4. N. A. Hagen, M. W. Kudenov, "Review of snapshot spectral imaging technologies," Opt. Eng. 52(9) 090901 (23 September 2013).

5. J. Tanida, T. Kumagai, K. Yamada, S. Miyatake, K. Ishida, T. Morimoto, N. Kondou, D. Miyazaki, and Y. Ichioka, "Thin observation module by bound optics (TOMBO): concept and experimental verification," Appl. Opt. 40, 1806-1813 (2001).

6. A. Portnoy, N. Pitsianis, X. Sun, D. Brady, R. Gibbons, A. Silver, R. Te Kolste, C. Chen, T. Dillon, and D. Prather, "Design and characterization of thin multiple aperture infrared cameras," Appl. Opt. 48, 2115-2126 (2009).

7. F. de la Barrière, G. Druart, N. Guérineau and J. Taboury, "Design strategies to simplify and miniaturize imaging systems," Appl. Opt. 50, 943, (2011).

8. G. Carles, G. Muyo, N. Bustin, A. Wood, and A. R. Harvey, "Compact multiaperture imaging with high angular resolution," J. Opt. Soc. Am. A 32, 411 419 (2015).

9. G. Druart, N. Guérineau, R. Haïdar, E. Lambert, M. Tauvy, S. Thétas, S. Rommeluère, J. Primot and J. Deschamps, "MULTICAM: a miniature cryogenic camera for infrared detection," Proc. SPIE. 6992, 69920G, (2008).

10. K. Cossu, G. Druart, E. Belhaire, F. Champagnat, T. Lépine, "Design, implementation and performance analysis of cooled infrared cameras with single FPA depth estimation capabilities," Proc. SPIE 9948 99480B-1 (2016).

11. R. Horstmeyer, G. Euliss, R. Athale, and M. Levoy, "Flexible multimodal camera using a light field architecture," Proceedings of the IEEE International Conference on Computational Photography (IEEE, 2009).

12. A. Manakov, J. F. Restrepo, O. Klehm, R. Hegedus, E. Eisemann, H. Seidel, and I. Ihrke, "A reconfigurable camera add-on for high dynamic range, multispectral, polarization, and light-field imaging," ACM Trans. Graph. 32, 47:1-47:14 (2013).

13. S. Pacheco, and R. Liang, "Snapshot, reconfigurable multispectral and multi-polarization telecentric imaging system," Opt. Express 22, 1637716385 (2014). 